\title{
Performed Medical History Result End Relative To Reference Code
}

National Cancer Institute

\section{Source}

National Cancer Institute. Performed Medical History Result End Relative To Reference

Code. NCl Thesaurus. Code C93928.

A coded value specifying the end of the medical history event with respect to the sponsor-defined reference period. 